\title{
The Correlation of Lung Function Indexes and Survival Time of Patients with Advanced Lung Cancer
}

\author{
Wenjing Xu ${ }^{1}$, Zhenghua Jiang ${ }^{1 *}$, Dongyun Huang ${ }^{2}$, Muyun Zhu ${ }^{1}$, Qian Huang ${ }^{1}$, Hui Ge ${ }^{1}$, Juan Liu ${ }^{1}$, \\ Yan Qin ${ }^{1}$ \\ ${ }^{1}$ Department of Pulmonary Medicine, Subei People's Hospital, Yangzhou, China; ${ }^{2}$ Department of Geriatric Medicine, Subei People's \\ Hospital, Yangzhou, China. \\ Email: *xwj0817@sina.com
}

Received November $14^{\text {th }}, 2012$; revised December $16^{\text {th }}, 2012$; accepted December $24^{\text {th }}, 2012$

\begin{abstract}
Background: To those patients with advanced lung cancer, the ultimate objective is to improve the quality of life, and lung function is an important factor affecting quality of life. We detect lung function of patients with lung cancer and study the correlation between lung function and the patients' survival time, to provide reference for evaluation of disease progression and prognosis. Methods: Lung function was detected on 59 cases of lung cancer and 63 normal controls. The relationship between lung function indexes and survival time was analyzed. Results: There was significant difference in ventilation function and diffusing capacity between lung cancer group and control group. Vital capacity (VC), Forced expiratory volume in one second (FEV1), Forced vital capacity (FVC), peak expiratory flow (PEF), peak expiratory flow\% (PEF\%), Maximal ventilatory volume (MVV) were positively correlated to survival time in patients with advanced lung cancer $(\mathrm{r}=0.28522064,0.28053851,0.28289252,0.26908133,0.26335034,0.28409036, \mathrm{P}<0.05)$, residual volume/total lung capacity was negatively correlated to survival time $(\mathrm{r}=-0.30760097, \mathrm{P}<0.05)$. Conclusions: The lung function decrease in the patients with lung cancer. Vital capacity (VC), Forced expiratory volume in one second (FEV1), Forced vital capacity (FVC), peak expiratory flow (PEF), peak expiratory flow\% (PEF\%), Maximal ventilatory volume (MVV), and residual volume/total lung capacity are correlated to survival time in patients with advanced lung cancer. The lung function indexes are important marker of prognosis of patients with lung cancer.
\end{abstract}

Keywords: Lung Function Indexes; Survival Time; Lung Cancer

\section{Introduction}

Lung cancer is the leading cause of cancer death among men and women worldwide, and most of them have locally advanced or metastatic disease at diagnosis which is unsuitable for surgery [1]. To those patients, the ultimate objective is to prolong survival time without leading to deterioration in quality of life, and the improvement of the quality of life has wan more and more attention in recent years. As we all know that the quality of life is closely related to lung function for patients with chronic lung diseases including lung cancer. Based on this theory, we hope to understand the lung function change and the correlation between lung function and survival time of patients with advanced lung cancer of patients with advanced lung cancer. We detected typical lung function indexes of 59 cases of lung cancer and compared with 63 normal controls, to provide reference for evaluation of disease progression and prognosis.

"Corresponding author.

\section{Patients and Methods}

Patients: We selected 59 hospitalized patients with lung cancer in our hospital from January 2009 to October 2011, all the cases were histologically proven as primitive non small cell lung cancer by transbronchoscopic biopsy or CT guided lung biopsy, including 52 male cases and 7 female cases, age from 40 to 77 years old, mean age is $64.27+/-8.74$ years old, 28 cases of adenocarcinoma, 30 cases of squamous cell cancer, and one case of adenosquamous carcinoma, all patients underwent chest CT, head MRI, B ultrasound, and ECT examination, according to the TNM stage criteria, all of the cases were diagnosed as advanced lung cancer (stage IIIB or IV). Normal control group: 63 cases of healthy subjects, including 51 male cases, 12 female cases, aged from 41 to 88 years old, the average age is $56.76+/-8.90$ years. All patients provided written informed consent, and the protocol was approved by the institutional review board in agreement with local regulatory requirements.

Methods: Lung function tests of patients before treat- 
ment and healthy control were performed using the Spirometer System (Jager, Germany). The index including cases' height, weight, sex, age, and temperature, atmospheric pressure etc. were input before the detection, and the corresponding predictive value were generated automatically by the computer. All the cases underwent the vital capacity (VC), forced expiratory volume in 1 second (FEV1), forced vital capacity (FVC), forced expiratory volume in 1 second/forced vital capacity (FEV1/ FVC), maximal ventilatory volume (MVV), residual volume/total lung capacity (RV/TLC) and diffusing capacity of the lung for carbon monoxide (DLCO), diffusion coefficient (DLCO/VA) detection. The results were demonstrated by the percent of reality value on predictive value.

Statistical Analysis: All the results were statistically demonstrated by $\mathrm{X} \pm \mathrm{S}$, using PEMS3.1 software to process data, and the comparison of two samples' mean were detected by $\mathrm{T}$ test. $\mathrm{X}^{2}$ statistic was applied to test the linear relationship between the lung funcion indexes and survival time.

\section{Results}

The comparison of lung functional parameters between lung cancer group and normal control group

As shown in Table 1, in lung cancer group, RV and RV/TCL index are higher than those of normal control group, while the former with no statistically significant difference and the latter with statistically significant difference; and the other parameters are lower than those of normal control group with statistically significant difference.

As shown in Table 2, in lung cancer group, the small airway function indexes of $\mathrm{V} 25, \mathrm{~V} 25 \%$, V $50, \mathrm{~V} 50 \%$ were significantly lower than those in normal control group with statistically significant difference.

As shown in Table 3, in the lung cancer group, lung diffusion function indicators were significantly lower than those in normal control group with statistically significant difference.

Table 1. Pulmonary ventilation function test results of lung cancer group and normal control group.

\begin{tabular}{|c|c|c|c|c|c|c|c|c|c|c|c|}
\hline Lung functional parameters & \multicolumn{2}{|l|}{$\mathrm{VC}$} & \multicolumn{2}{|c|}{$\mathrm{VC} \%$} & \multicolumn{2}{|c|}{ FEV1 } & \multicolumn{2}{|c|}{ FEV $1 \%$} & \multicolumn{2}{|r|}{$\mathrm{FVC}$} & $\mathrm{FVC} \%$ \\
\hline Normal control group 63 cases & \multicolumn{2}{|c|}{$3.74 \pm 0.73$} & \multicolumn{2}{|c|}{$103.24 \pm 14.85$} & \multicolumn{2}{|c|}{$3.27 \pm 0.68$} & \multicolumn{2}{|c|}{$112.66 \pm 14.52$} & \multicolumn{2}{|c|}{$3.64 \pm 0.74$} & $101.51 \pm 18.42$ \\
\hline Lung cancer group 59 cases & \multicolumn{2}{|c|}{$2.93 \pm 0.72$} & \multicolumn{2}{|c|}{$82.29 \pm 17.86$} & \multicolumn{2}{|c|}{$2.12 \pm 0.65$} & \multicolumn{2}{|c|}{$77.03 \pm 20.73$} & \multicolumn{2}{|c|}{$2.90 \pm 0.73$} & $83.68 \pm 17.99$ \\
\hline $\mathrm{T}$ value & \multicolumn{2}{|l|}{6.1799} & \multicolumn{2}{|c|}{7.0629} & \multicolumn{2}{|c|}{9.5279} & \multicolumn{2}{|c|}{11.0524} & \multicolumn{2}{|c|}{5.5345} & 5.4018 \\
\hline$P$ value & \multicolumn{2}{|l|}{0.0000} & \multicolumn{2}{|c|}{0.0000} & \multicolumn{2}{|c|}{0.0000} & \multicolumn{2}{|c|}{0.0000} & \multicolumn{2}{|c|}{0.0000} & 0.0000 \\
\hline Lung functional parameters & FEV1/FVC\% & & $\mathrm{EF}$ & $\mathrm{PE}$ & & & & MV & & RV & $\mathrm{RV} / \mathrm{TCL}$ \\
\hline Normal control group 63 cases & $90.02 \pm 4.57$ & 7.8 & \pm 1.87 & 104.64 & 22.59 & 117.32 & 30.67 & $108.99=$ & 5.84 & $1.99 \pm 0.44$ & $35.26 \pm 7.22$ \\
\hline Normal control group 63 cases & $72.73 \pm 11.44$ & 5.7 & \pm 1.80 & $77.51=$ & 2.80 & 66.82 & 20.48 & $65.47 \pm$ & 7.96 & $2.29 \pm 3.53$ & $40.20 \pm 9.533$ \\
\hline $\mathrm{T}$ value & 11.0922 & & 976 & 6.6 & & 9.9 & & 10.7 & & 0.6657 & 3.2433 \\
\hline
\end{tabular}

Table 2. Small airway function test results of lung cancer group and normal control group.

\begin{tabular}{lccc}
\hline Lung functional parameters & V25 & V25\% & V50 \\
\hline Normal control group 63 cases & $7.27 \pm 1.88$ & $110.64 \pm 25.66$ & $4.43 \pm 1.56$ \\
Normal control group 63 cases & $4.29 \pm 1.96$ & $65.45 \pm 29.82$ & $2.03 \pm 1.18$ \\
T value & 8.5820 & 8.9893 & 9.5301 \\
P value & 0.0000 & 0.0000 & 0.0000 \\
\hline
\end{tabular}

Table 3. Lung diffusion function test results of lung cancer group and normal control group.

\begin{tabular}{lcccc}
\hline Lung functional parameters & DLCO & DLCO $\%$ & DLCO/VA & DLCO/VA\% \\
\hline Normal control group 63 cases & $8.42 \pm 1.85$ & $97.25 \pm 13.59$ & $1.51 \pm 0.2$ & $101.48 \pm 13.72$ \\
Normal control group 63 cases & $5.57 \pm 1.86$ & $68.21 \pm 22.09$ & $1.26 \pm 0.36$ & $91.09 \pm 22.42$ \\
T value & 8.4741 & 8.8082 & 4.9179 & 3.1092 \\
P value & 0.0000 & 0.0000 & 0.0000 & 0.0023 \\
\hline
\end{tabular}


The correlation of lung function indexes and survival time of patients with lung cancer

As shown in Table 4, all of the 59 patients in lung cancer group were followed up. The survival time was calculated from the day of final diagnosis to patients' death or the last follow-up time (January 23, 2012). The follow-up of patients showed that the survival period was 122 - 936 days and the median survival time was 357 days. Pulmonary function indexes including VC, FEV1, FVC, PEF, PEF\%, MVV were positively correlated with patients' survival (correlation coefficient were 0.28522064, $0.28053851,0.28289252,0.26908133,0.26335034$ and 0.28409036 respectively, $\mathrm{P}<0.05$ ), while $\mathrm{RV} / \mathrm{TCL}$ ratio was negatively correlated with the survival period of patients(correlation coefficient $=-0.30760097, \mathrm{P}<0.05$ ).

\section{Discussion}

Lung cancer is one of the most common malignant tumors which severely threat human health, the morbility is

Table 4. The coefficient correlation of lung function indexes and survival time of patients with lung cancer.

\begin{tabular}{|c|c|c|c|}
\hline $\begin{array}{l}\text { Lung function } \\
\text { indexes }\end{array}$ & $\begin{array}{l}\text { Coefficient } \\
\text { correlation }\end{array}$ & $\mathrm{T}$ value & $P$ value \\
\hline $\mathrm{VC}$ & 0.28522064 & 2.2467 & 0.0285 \\
\hline $\mathrm{VC} \%$ & 0.21324802 & 1.6479 & 0.1049 \\
\hline FEV1 & 0.28053851 & 2.2066 & 0.0314 \\
\hline FEV1\% & 0.22119043 & 1.7124 & 0.0923 \\
\hline $\mathrm{FVC}$ & 0.28289252 & 2.2268 & 0.0299 \\
\hline $\mathrm{FVC} \%$ & 0.21979086 & 1.7010 & 0.0944 \\
\hline FEV1/FVC\% & 0.10095762 & 0.7661 & 0.4468 \\
\hline PEF & 0.26908133 & 2.1093 & 0.0393 \\
\hline PEF\% & 0.26335034 & 2.0610 & 0.0439 \\
\hline V25 & 0.16445738 & 1.2588 & 0.2132 \\
\hline $\mathrm{V} 25 \%$ & 0.16143446 & 1.2350 & 0.2219 \\
\hline V50 & 0.18455800 & 1.4177 & 0.1617 \\
\hline V $50 \%$ & 0.17189632 & 1.3174 & 0.1930 \\
\hline MVV & 0.28409036 & 2.2370 & 0.0292 \\
\hline MVV\% & 0.24133313 & 1.8775 & 0.0656 \\
\hline RV & 0.08972369 & 0.6801 & 0.4992 \\
\hline $\mathrm{RV} / \mathrm{TCL}$ & -0.30760097 & -2.4407 & 0.0178 \\
\hline DLCO & 0.20516108 & 1.5826 & 0.1190 \\
\hline DLCO $\%$ & 0.12343208 & 0.9391 & 0.3517 \\
\hline DLCO/VA & 0.18542021 & 1.4246 & 0.1597 \\
\hline DLCO/VA\% & 0.13735532 & 1.0469 & 0.2995 \\
\hline
\end{tabular}

rising year by year, and there are more than 1 million new cases emerged each year [2]. The cure rate of lung cancer is low, according to the statistics, the five year survival rate of lung cancer is only about $20 \%$, and the main reason is most of the new cases were diagnosed in the advanced stage with remote metastasis while only $15 \%$ of the patients were diagnosed in the early stage [1].

It is well know that lung function is an important prognostic factor for patients with early stage lung cancer who considering surgery $[3,4]$, but for those patients with advanced lung cancer the prognosis assessment of lung function has not been applied extensively.

We enrolled 59 hospitalized patients for this study, through detecting different lung function indexes and comparing with nomal control, we try to find the importance of lung function on the proganosis of these patients. The results of our study show that in lung cancer group, $\mathrm{RV}$ and RV/TCL index are higher than those of normal control group, and the other pulmonary ventilation function indexes are lower than those of normal control group with statistically significant difference. Also we found the small airway function indexes of V25, V25\%, V50, $\mathrm{V} 50 \%$ in lung cancer group were significantly lower than those in normal control group with statistically significant difference. These data indicate that patients with lung cancer are complicated with obstructive ventilatory disorder, which is consistent with the relevant studies.

The mechanism of lung ventilation function disorder and small airway dysfunction in patients with lung cancer may be as follows: To begin with, most patients had long-term smoking history, and part of them had chronic obstructive pulmonary disease and pulmonary emphysema as well, thereby causing obstructive ventilatory impairment [5]. Secondly, lung masses, atelectasis, obstructive pneumonia and other factors related with lung cancer may lead to the reduction of ventilated lung tissue, thereby causing ventilatory impairment. Thirdly, due to tumor factors, the secretion of prostaglandin increased [6], causing bronchiospasm or part of alveolar closure, resulting in airway obstruction.

Diffusion function is an important part of the pulmonary gas exchange function, through diffusion $\mathrm{O}_{2}$ move from the alveoli into the pulmonary capillaries, and $\mathrm{CO}_{2}$ discharged from pulmonary capillaries into the alveolar. Margaritora et al. [7,8] confirmed that DLCO was an effective predictive factor of pulmonary complications for patients with lung cancer. Baser et al. [9] indicated that preoperative low levels of DLCO $(<45 \%)$ in patients with lung cancer was related to the increased morbidity of postoperative pulmonary complications and increased mortality. In our study, we found that lung diffusion function indicators in lung cancer group were significantly lower than those in normal control group with statistically significant difference, which mean that lung 
cancer may cause the decline of diffusion function. The mechanism for this may as follows: Firstly, the obstructive pneumonia, atelectasis and pleural diseases related with lung cancer can cause the reduction of lung volume, limitation of ventilation function and reduction of diffusion area, at the same time, the compensatory of surrounding lung tissue can increase the ventilation, thus cause the ventilation/perfusion imbalance [10,11]; Secondly, the blood vessels around the tumor may be blocked due to the mechanic compression, and the reduction of blood flow may increase the ventilation/perfusion ratio; Thirdly, the lung cancer cells may be transferred via the blood stream, blocking small vessels and leading to ventilation/perfusion ratio further disorder [12].

Our study also found that Pulmonary function indexes including VC, FEV1, FVC, PEF, PEF\%, MVV were positively correlated with patients' survival while RV/ TCL ratio was negatively correlated with the survival period of patients. It means that the decreased VC, FEV1, FVC, PEF, PEF\%, MVV and increased RV/TCL are high risk factors for patients with lung cancer.

\section{Conclusion}

Both the ventilation function and diffusion function obviously decreased in patients with advanced lung cancer, and some of the lung function indexes including $\mathrm{VC}$, FEV1, FVC, PEF, PEF\%, MVV, and RV/TCL can be used as important factors for estimating the prognosis of patients with lung caner. We all know that is impossible for us to radically cure patients with advanced lung cancer so far, but through all kinds of treatments we try to make lung cancer as a chronic disease, which means patient can live with tumor for a long time. Good lung function is important to those patients to keep a good quality of life, and rehabilitation may be needed to improve the lung function of patients with advanced lung cancer [13].

\section{REFERENCES}

[1] A. Jemal, R. Siegel, E. Ward, et al., "Cancer Statistics, 2009," CA: A Cancer Journal for Clinicians, Vol. 59, No. 4, 2009, pp. 225-249. doi:10.3322/caac.20006

[2] J. Ferlay, H. R. Shin, F. Bray, et al., "Estimates of Worldwide Burden of Cancer in 2008: GLOBOCAN 2008," International Journal of Cancer, Vol. 127, No. 12, 2010, pp. 2893-2917.doi:10.1002/ijc.25516

[3] T. Iizasa, M. Suzuki, K. Yasufuku, et al., "Preoperative Pulmonary Function as a Prognostic Factor for Stage I
Non-Small Cell Lung Carcinoma," The Annals of Thoracic Surgery, Vol. 77, No. 6, 2004, pp. 1896-1902.

[4] T. Win, A. Jackson, L. Sharples, et al., "Relationship between Pulmonary Function and Lung Cancer Surgical Outcome," European Respiratory Journal, Vol. 25, No. 4, 2005, pp. 594-599. doi:10.1183/09031936.05.00077504

[5] S. Raviv, K. A. Hawkins, M. M. DeCamp Jr., et al., "Lung Cancer in Chronic Obstructive Pulmonary Disease Enhancing Surgical Options and Outcomes," American Journal of Respiratory and Critical Care Medicine, Vol. 183, No. 9, 2011, pp. 1138-1146. doi:10.1164/rccm.201008-1274CI

[6] K. Yoshimatsu, N. K. Altorki, D. Golijanin, et al., "Inducible Prostaglandin E Synthase is Overexpressed in Non-Small Cell Lung Cancer," Clinical Cancer Research, Vol. 7, No. 9, 2001, pp. 2669-2674.

[7] S. Margaritora, A. Cesario, G. Gusumano, et al., "Is Pulmonary Function Damaged by Neoadjuvant Lung Cancer Therapy? A Comprehensive Serial Time-Trend Analysis of Pulmonary Function after Induction Radiochemotherapy Plus Surgery," The Journal of Thoracic and Cardiovascular Surgery, Vol. 139, No. 6, 2010, pp. 1457-1463. doi:10.1016/j.jtcvs.2009.10.023

[8] S. Takeda, Y. Funakoshi, Y. Kadota, et al., "Fall in Diffusing Capacity Associated with Induction Therapy for Lung Cancer: A Predictor of Postoperative Complication? The Annals of Thoracic Surgery, Vol. 82, No. 1, 2006, pp. 232-236. doi:10.1016/j.athoracsur.2006.01.045

[9] S. Baser, V. R. Shannon, G. A. Eapen, et al., "Smoking Cessation after Diagnosis of Lung Cancer Is Associated with a Beneficial Effect on Performance Status," Chest, Vol. 130, No. 6, 2006, pp. 1784-1790.

[10] K. L. Tsai, E. Gupta and L. B. Haramati, "Pulmonary Atelectasis: A Frequent Alternative Diagnosis in Patients Undergoing CT-PA for Suspected Pulmonary Embolism," Emergency Radiology, Vol. 10, No. 5, 2004, pp. 282-286. doi:10.1007/s10140-004-0328-5

[11] A. G. Agustí, J. Cardús, J. Roca, et al., "Ventilation-Perfusion Mismatch in Patients with Pleural Effusion: Effects of Thoracentesis," American Journal of Respiratory and Critical Care Medicine, Vol. 156, No. 4, 1997, pp. 1205-1209.

[12] M. Reyhan, M. Aydin, A. Sukan, et al., "VentilationPerfusion Mismatch Secondary to Arterial Bullet Embolism," Clinical Nuclear Medicine, Vol. 32, No. 4, 2007, pp. 330-332. doi:10.1097/01.rlu.0000256856.53656.b1

[13] A. L. Cheville, A. M. Dose, J. R. Basford, et al., "Insights into the Reluctance of Patients with Late-Stage Cancer to Adopt Exercise as a Means to Reduce Their Symptoms and Improve Their Function," Journal of Pain and Symptom Management, Vol. 44, No. 1, 2012, pp. 84-94. doi:10.1016/j.jpainsymman.2011.08.009 